TRANSACTIONS OF THE

AMERICAN MATHEMATICAL SOCIETY

Volume 361, Number 8, August 2009, Pages 4247-4267

S 0002-9947(09)04697-2

Article electronically published on March 13, 2009

\title{
EVERY SUM SYSTEM IS DIVISIBLE
}

\author{
MASAKI IZUMI
}

\begin{abstract}
We show that every sum system is divisible. Combined with B. V. R. Bhat and R. Srinivasan's result, this shows that every product system arising from a sum system (and every generalized CCR flow) is either of type I or type III. A necessary and sufficient condition for such a product system to be of type I is obtained.
\end{abstract}

\section{INTRODUCTION}

An $E_{0}$-semigroup is a weakly continuous semigroup of unital *-endomorphisms on $\mathbb{B}(H)$, the algebra of all bounded operators on a separable infinite dimensional Hilbert space $H$. W. Arveson [2] introduced the notion of a product system (a continuous tensor product system of Hilbert spaces) and showed that the product system associated with an $E_{0}$-semigroup completely determines the cocycle conjugacy class of the $E_{0}$-semigroup. On the other hand, Arveson [3] also showed that every product system arises from an $E_{0}$-semigroup (see also [9], 12]).

$E_{0}$-semigroups (and hence product systems) are classified into three categories, type I, II, and III. Using a quasi-free representation of the CAR (canonical anticommutation relation) algebras, R. Powers [11] constructed the first example of an $E_{0}$-semigroup of type III. It is technically very difficult to construct such an example, and one had to wait for more than 10 years before B. Tsirelson [13] produced uncountably many mutually non-isomorphic product systems of type III. His construction uses continuous sums of Hilbert spaces coming from off-white noise.

Recently, a few attempts [5], [7, 8] were made to understand Tsirelson's construction from the viewpoint of functional analysis. Bhat and Srinivasan 5 introduced the notion of a sum system, which is an axiomatization of Tsirelson's continuous sum of Hilbert spaces. A sum system gives rise to a product system via the second quantization procedure using the CCR (canonical commutation relation). Among others, a dichotomy result about types was proved in [5], which says that every product system arising from a divisible sum system is either of type I or of type III. A sum system is said to be divisible if it has sufficiently many real and imaginary addits (called additive units in [5]). While Bhat and Srinivasan 5 dealt with product systems, the present author [7 directly gave a description of Tsirelson's $E_{0}$-semigroups in terms of perturbations of the shift semigroup of $L^{2}(0, \infty)$ and the CCR algebras.

Received by the editors August 14, 2007.

2000 Mathematics Subject Classification. Primary 46L55, 47D03, 81S05.

Key words and phrases. $E_{0}$-semigroups, product system, type I, type III.

This work was supported by JSPS.

(C)2009 American Mathematical Society Reverts to public domain 28 years from publication 
As a consequence of the two approaches [5] and [7, a class of $E_{0}$-semigroups, called generalized CCR flows, was introduced in [8]. A generalized CCR flow is constructed from a pair of $C_{0}$-semigroups acting on a real Hilbert space on the one hand, and on the other hand, the product system associated with it arises from a sum system. In [8], we constructed continuously many mutually non-cocycle conjugate generalized CCR flows of type III different from Tsirelson's examples.

The main purpose of this paper is to show that every sum system is divisible. Combined with the above-mentioned dichotomy result, this implies that every product system arising from a sum system (and every generalized CCR flow) is either of type I or type III. In our proof, we carefully analyze the domains of the generators of the two $C_{0}$-semigroups giving the generalized CCR flows, whose product system arises from the given sum system. In [8], a type I criterion was given for a divisible sum system of finite index. Our main theorem shows that the spaces of real and imaginary addits have nice subspaces with manageable topologies, which enables us to prove the type I criterion in full generality.

\section{Preliminaries and notation}

In this section we fix the notation used in this paper. For an operator $A$, we denote the range of $A$ by $\operatorname{Ran}(A)$, and the kernel of $A$ by $\operatorname{Ker}(A)$. We denote the identity operator on a Hilbert space $H$ by $I_{H}$ (or simply by $I$ if no confusion arises). Our inner product is linear in the first variable.

2.1. $E_{0}$-semigroups and product systems. For $E_{0}$-semigroups, our basic reference is [4].

Definition 2.1. A product system of Hilbert spaces is a one-parameter family of separable complex Hilbert spaces $\left\{H_{t} ; t \geq 0\right\}$, together with unitary operators $U_{s, t}: H_{s} \otimes H_{t} \rightarrow H_{s+t}$ for $s, t \in(0, \infty)$, satisfying the following two axioms:

(1) (Associativity) For any $s_{1}, s_{2}, s_{3} \in(0, \infty)$,

$$
U_{s_{1}, s_{2}+s_{3}}\left(I_{H_{s_{1}}} \otimes U_{s_{2}, s_{3}}\right)=U_{s_{1}+s_{2}, s_{3}}\left(U_{s_{1}, s_{2}} \otimes I_{H_{s_{3}}}\right) .
$$

(2) (Measurability) There exists a countable set $H^{0}$ of sections $\mathbb{R} \ni t \rightarrow h_{t} \in H_{t}$ such that $t \mapsto\left\langle h_{t}, h_{t}^{\prime}\right\rangle$ is measurable for any two $h, h^{\prime} \in H^{0}$, and the set $\left\{h_{t} ; h \in H^{0}\right\}$ is total in $H_{t}$, for each $t \in(0, \infty)$. Further it is also assumed that the map $(s, t) \mapsto\left\langle U_{s, t}\left(h_{s} \otimes h_{t}\right), h_{s+t}^{\prime}\right\rangle$ is measurable for any two $h, h^{\prime} \in H^{0}$.

Two product systems $\left(\left\{H_{t}\right\},\left\{U_{s, t}\right\}\right)$ and $\left(\left\{H_{t}^{\prime}\right\},\left\{U_{s, t}^{\prime}\right\}\right)$ are said to be isomorphic if there exists a unitary operator $V_{t}: H_{t} \rightarrow H_{t}^{\prime}$, for each $t \in(0, \infty)$ satisfying

$$
V_{s+t} U_{s, t}=U_{s, t}^{\prime}\left(V_{s} \otimes V_{t}\right) .
$$

The above definition is slightly different from Arveson's original one though the two definitions are equivalent, of course. Note that we need not consider measurable structures while dealing with isomorphisms of product systems (see [9]).

For an $E_{0}$-semigroup $\left\{\alpha_{t}\right\}_{t \geq 0}$, let $H_{t}$ be the space of intertwiners between the identity of $H$ and $\alpha_{t}$, that is,

$$
H_{t}=\left\{T \in \mathbb{B}(H) ; \alpha_{t}(X) T=T X, \forall X \in \mathbb{B}(H)\right\} .
$$

Then $H_{t}$ is a Hilbert space with inner product $\langle S, T\rangle I_{H}=T^{*} S$. Identifying $x_{1} \otimes x_{2}$ with $x_{1} x_{2}$ for $x_{1} \in H_{t}$ and $x_{2} \in H_{s}$, one can see that the family $\left\{H_{t}\right\}_{t}$ satisfies the axiom of a product system. 
Definition 2.2. A unit for a product system is a non-zero section $\left\{u_{t} ; t \geq 0\right\}$, such that the map $t \mapsto\left\langle u_{t}, h_{t}\right\rangle$ is measurable for any $h \in H^{0}$ and

$$
U_{s, t}\left(u_{s} \otimes u_{t}\right)=u_{s+t}, \forall s, t \in(0, \infty) .
$$

A product system (an $E_{0}$-semigroup) is said to be of type I if units exist for the product system and they generate the product system (see [2, 4] for the precise meaning). It is of type II if units exist but they do not generate the product system. It is of type III (or unitless) if there is no unit.

2.2. Symmetric Fock space. For a complex Hilbert space $K$, we denote by $\Gamma(K)$ the symmetric Fock space associated with $K$, and by $\Phi$ the vacuum vector in $\Gamma(K)$ (see [10]). For any $x \in K$, the exponential vector of $x$ is defined by

$$
e(x)=\bigoplus_{n=0}^{\infty} \frac{1}{\sqrt{n !}} x^{\otimes^{n}}
$$

where $x^{\otimes^{0}}=\Phi$. Then the set of all exponential vectors $\{e(x): x \in K\}$ is a linearly independent total set in $\Gamma(K)$. For a unitary $U \in \mathbb{B}(K)$, we denote by $\operatorname{Exp}(U)$ the unitary in $\mathbb{B}(\Gamma(K))$ given by $\operatorname{Exp}(U) e(x)=e(U x)$. The Weyl operator $W(x)$ for $x \in K$ is a unitary operator of $\Gamma(K)$ determined by

$$
W(x)(e(y))=e^{-\frac{\|x\|^{2}}{2}-\langle y, x\rangle} e(y+x) .
$$

The Weyl operators satisfy the relation $W(x) W(y)=e^{i \operatorname{Im}\langle x, y\rangle} W(x+y)$. The *-algebra generated by $\{W(x)\}_{x \in K}$ is called the Weyl algebra for $K$.

For a real Hilbert space $G$, we denote the complexification of $G$ by $G^{\mathbb{C}}$. For two Hilbert spaces $G_{1}, G_{2}$, we denote by $\mathcal{S}\left(G_{1}, G_{2}\right)$ the set of bounded invertible operators $A$ in $\mathbb{B}\left(G_{1}, G_{2}\right)$ such that $I-A^{*} A$ is a Hilbert-Schmidt operator. An operator in $\mathcal{S}\left(G_{I}, G_{2}\right)$ is called an equivalence operator. In the above definition and elsewhere, by invertibility we mean that the inverse is also bounded.

For two real Hilbert spaces $G_{1}, G_{2}$ and $A \in \mathcal{S}\left(G_{1}, G_{2}\right)$, define a real linear operator $S_{A}: G_{1}^{\mathbb{C}} \rightarrow G_{2}^{\mathbb{C}}$ by $S_{A}(u+i v)=A u+i\left(A^{-1}\right)^{*} v$ for $u, v \in G_{1}$. Then $S_{A}$ is a symplectic isomorphism between $G_{1}^{\mathbb{C}}$ and $G_{2}^{\mathbb{C}}$ (i.e. $S_{A}$ is a real linear, bounded, invertible map satisfying $\operatorname{Im}\left(\left\langle S_{A} x, S_{A} y\right\rangle\right)=\operatorname{Im}\langle x, y\rangle$ for all $x, y \in G_{1}^{\mathbb{C}}$; see [10, page $162])$. In general $S_{A}$ is not complex linear, unless $A$ is unitary.

The following theorem, a generalization of Shale's theorem, is used to construct the product system from a sum system in [5].

Theorem 2.3. Let $G_{1}$ and $G_{2}$ be real Hilbert spaces and $A \in \mathcal{S}\left(G_{1}, G_{2}\right)$. Then there exists a unique unitary operator $\Gamma(A): \Gamma\left(G_{1}^{\mathbb{C}}\right) \rightarrow \Gamma\left(G_{2}^{\mathbb{C}}\right)$ satisfying

$$
\Gamma(A) W(z) \Gamma(A)^{*}=W\left(S_{A} z\right), \quad \forall z \in G_{1}^{\mathbb{C}},
$$

with the normalization condition $\left\langle\Gamma(A) \Phi_{1}, \Phi_{2}\right\rangle \in \mathbb{R}^{+}$, where $\Phi_{1}$ and $\Phi_{2}$ are the vacuum vectors in $\Gamma\left(G_{1}^{\mathbb{C}}\right)$ and $\Gamma\left(G_{2}^{\mathbb{C}}\right)$ respectively. If moreover $G_{3}$ is a real Hilbert space and $B \in \mathcal{S}\left(G_{2}, G_{3}\right)$, then $\Gamma(B A)=\Gamma(B) \Gamma(A)$ holds.

Note that $\Gamma\left(A^{-1}\right)=\Gamma(A)^{*}$ automatically holds from the above statement.

The next lemma follows from the proof of [8, Lemma 4.6]:

Lemma 2.4. Let $G_{1}$ and $G_{2}$ be real Hilbert spaces and let $R_{0}$ be a real linear operator from $G_{1}^{\mathbb{C}}$ to $G_{2}^{\mathbb{C}}$ with dense domain $D\left(R_{0}\right)$, which preserves imaginary parts of the inner product. Suppose that there exists a unitary operator 
$U \in \mathbb{B}\left(\Gamma\left(G_{1}^{\mathbb{C}}\right), \Gamma\left(G_{2}^{\mathbb{C}}\right)\right)$ satisfying

$$
U W(x) U^{*}=W\left(R_{0} x\right), \forall x \in D\left(R_{0}\right) .
$$

Then $R_{0}$ extends to a bounded invertible operator $R$ from $G_{1}^{\mathbb{C}}$ onto $G_{2}^{\mathbb{C}}$. Further it is true that $R \in \mathcal{S}\left(G_{1} \oplus G_{1}, G_{2} \oplus G_{2}\right)$, where we identify $G_{i} \oplus G_{i}$ with $G_{i}^{\mathbb{C}}$ equipped with the real inner product $\langle\cdot, \cdot\rangle_{\mathbb{R}}=\operatorname{Re}\langle\cdot, \cdot\rangle$.

2.3. Sum systems. Next we define the notion of a sum system.

Definition 2.5. A sum system is a two-parameter family $\left\{G_{s, t}\right\}_{0 \leq s<t \leq \infty}$ of closed subspaces of a real Hilbert space $G_{0, \infty}$ satisfying the inclusion relations $G_{s, t} \subset G_{s^{\prime}, t^{\prime}}$ for all $(s, t) \subset\left(s^{\prime}, t^{\prime}\right)$ together with a $C_{0}$-semigroup $\left\{S_{t}\right\}_{t \geq 0}$ acting on $G_{0, \infty}$ such that the following hold for any $s \in(0, \infty)$ and $t \in(0, \infty]$ with $s<t$ :

(1) The restriction of $S_{s}$ to $G_{0, t}$ is in $\mathcal{S}\left(G_{0, t}, G_{s, s+t}\right)$.

(2) If $A_{s, t}: G_{0, s} \oplus G_{s, s+t} \mapsto G_{0, s+t}$, is the map $A_{s, t}(x \oplus y)=x+y$, for $x \in G_{0, s}$ and $y \in G_{s, s+t}$, then $A_{s, t} \in \mathcal{S}\left(G_{0, s} \oplus G_{s, s+t}, G_{0, s+t}\right)$.

We say that two sum systems $\left(\left\{G_{a, b}\right\},\left\{S_{t}\right\}\right)$ and $\left(\left\{G_{a, b}^{\prime}\right\},\left\{S_{t}^{\prime}\right\}\right)$ are isomorphic if there exists a family of equivalence operators $U_{t} \in \mathcal{S}\left(G_{0, t}, G_{0, t}^{\prime}\right)$ for $0<t<\infty$ preserving every structure of the sum systems, i.e., if $\left\{U_{t}\right\}$ satisfies

$$
A_{s, t}^{\prime}\left(\left.U_{s} \oplus S_{s}^{\prime}\right|_{G_{0, t}^{\prime}} U_{t}\right)=U_{s+t} A_{s, t}\left(\left.I_{G_{0, s}} \oplus S_{s}\right|_{G_{0, t}}\right),
$$

where $A_{s, t}^{\prime}$ is defined for $\left(\left\{G_{a, b}^{\prime}\right\},\left\{S_{t}^{\prime}\right\}\right)$ in a similar way to $A_{s, t}$ above.

The above definition, adopted in [8], is slightly stronger than the one given in 5.

Given a sum system $\left(\left\{G_{s, t}\right\},\left\{S_{t}\right\}\right)$, we define Hilbert spaces $H_{t}=\Gamma\left(G_{0, t}^{\mathbb{C}}\right)$, and unitary operators $U_{s, t}: H_{s} \otimes H_{t} \mapsto H_{s+t}$, by $U_{s, t}=\Gamma\left(A_{s, t}\right)\left(I_{H_{s}} \otimes \Gamma\left(\left.S_{s}\right|_{G_{0, t}}\right)\right)$. It is proved in [5] that $\left(\left\{H_{t}\right\},\left\{U_{s, t}\right\}\right)$ forms a product system. Isomorphic sum systems give rise to isomorphic product systems.

2.4. GCCR flows. Let $G$ be a real Hilbert space, and let $\left\{S_{t}\right\}$ be the shift semigroup of $L^{2}((0, \infty), G)$ (and of $\left.L^{2}\left((0, \infty), G^{\mathbb{C}}\right)\right)$ defined by

$$
\left(S_{t} f\right)(x)= \begin{cases}0 & (x<t), \\ f(x-t) & (t \leq x)\end{cases}
$$

for $f \in L^{2}((0, \infty), G)$. The CCR flow of index $\operatorname{dim} G$ is an $E_{0}$-semigroup $\alpha$ acting on $\mathbb{B}\left(\Gamma\left(L^{2}\left((0, \infty), G^{\mathbb{C}}\right)\right)\right)$, which is determined by $\alpha_{t}(W(f))=W\left(S_{t} f\right)$ for $f \in L^{2}\left((0, \infty), G^{\mathbb{C}}\right)$. The corresponding product system is the exponential product system of index $\operatorname{dim} G$ (see [3]), which is the simplest example of a product system arising from a sum system. Namely, let $G_{s, t}=L^{2}((s, t), G)$. Then the sum system $\left(\left\{G_{s, t}\right\},\left\{S_{t}\right\}\right)$, which we call the shift sum system of index $\operatorname{dim} K$, gives rise to the exponential sum system.

Since the CCR relation involves only the imaginary part of the inner product of the test functions, to construct an $E_{0}$-semigroup, we do not necessarily use the same time translation for both the real and imaginary test functions.

Definition 2.6. Let $\left\{S_{t}\right\}$ and $\left\{T_{t}\right\}$ be $C_{0}$-semigroups acting on a real Hilbert space $G$. We say that $\left\{T_{t}\right\}$ is a perturbation of $\left\{S_{t}\right\}$ if they satisfy

(1) $T_{t}{ }^{*} S_{t}=I$.

(2) $S_{t}-T_{t}$ is a Hilbert-Schmidt operator.

We call $\left(\left\{S_{t}\right\},\left\{T_{t}\right\}\right)$ as above a perturbation pair. 
Let $\left(\left\{S_{t}\right\},\left\{T_{t}\right\}\right)$ be a perturbation pair of $C_{0}$-semigroups acting on $G$. Then an easy application of well-known criteria ([1, [6]) shows the existence of the generalized CCR flow for $\left(\left\{S_{t}\right\},\left\{T_{t}\right\}\right)$ defined below (see [8, Lemma 2.3] for the proof).

Definition 2.7. Given a perturbation pair $\left(\left\{S_{t}\right\},\left\{T_{t}\right\}\right)$ of $C_{0}$-semigroups acting on a real Hilbert space $G$, we say that the $E_{0}$-semigroup $\left\{\alpha_{t}\right\}_{t \geq 0}$ acting on $\mathbb{B}\left(\Gamma\left(G^{\mathbb{C}}\right)\right)$ determined by

$$
\alpha_{t}(W(x+i y))=W\left(S_{t} x+i T_{t} y\right), \quad x, y \in G
$$

is a generalized CCR flow associated with the pair $\left\{S_{t}\right\}$ and $\left\{T_{t}\right\}$.

2.5. From sum systems to perturbation pairs. In the remaining two subsections, we recall the description of the $E_{0}$-semigroup associated with the product system constructed out of a sum system given in [8, Section 3].

Let $\left(\left\{G_{a, b}\right\},\left\{S_{t}\right\}\right)$ be a sum system. Denote $G=G_{0, \infty}, A_{t}=A_{t, \infty}$. We may consider $S_{t}$ as a bounded linear invertible map from $G$ onto $G_{t, \infty}$. Hence $\left(S_{t}^{*}\right)^{-1}$ is a well-defined bounded operator from $G$ onto $G_{t, \infty}$. When there is no possibility of confusion, we sometimes regard $\left(S_{t}^{*}\right)^{-1}$ as an element of $\mathbb{B}(G)$. Define $T_{t} \in \mathbb{B}(G)$ by

$$
T_{t}=\left(A_{t}^{*}\right)^{-1} A_{t}^{-1}\left(S_{t}^{*}\right)^{-1} \forall t \in[0, \infty) .
$$

Then $\left\{T_{t}\right\}$ forms a $C_{0}$-semigroup on $G$ ([8, Lemma 3.2]).

The following proposition is proved in [8, Proposition 3.3, Corollary 3.4]:

Proposition 2.8. Let $\left(\left\{G_{s, t}\right\},\left\{S_{t}\right\}\right)$ be a sum system, and let $\left\{T_{t}\right\}$ be the $C_{0^{-}}$ semigroup as above. Then $\left(\left\{S_{t}\right\},\left\{T_{t}\right\}\right)$ is a perturbation pair of $C_{0}$-semigroups. The product system for the generalized CCR flow for $\left(\left\{S_{t}\right\},\left\{T_{t}\right\}\right)$ is the one constructed out of the sum system $\left(\left\{G_{s, t}\right\},\left\{S_{t}\right\}\right)$.

We say that the above pair $\left(\left\{S_{t}\right\},\left\{T_{t}\right\}\right)$ of $C_{0}$-semigroups is associated with the sum system $\left(\left\{G_{a, b}\right\},\left\{S_{t}\right\}\right)$.

2.6. From perturbation pairs to sum systems. Let $G$ be a real Hilbert space, and let $\left(\left\{S_{t}\right\},\left\{T_{t}\right\}\right)$ be a perturbation pair of $C_{0}$-semigroups acting on $G$. We describe the product system for the generalized CCR flow associated with the pair $\left(\left\{S_{t}\right\},\left\{T_{t}\right\}\right)$.

Let $G_{0, t}=\operatorname{Ker}\left(T_{t}^{*}\right), G_{a, b}=S_{a}\left(G_{0, b-a}\right)$, and

$$
G_{0, \infty}=\overline{\bigcup_{t>0} G_{0, t}} .
$$

Let $P: G \rightarrow G_{0, \infty}$ be the orthogonal projection. We define $S_{t}^{0}$ and $T_{t}^{0}$ by

$$
S_{t}^{0}=P S_{t} P, \quad T_{t}^{0}=P T_{t} P .
$$

The following is [8, Proposition 3,6]:

Proposition 2.9. Let $G$ be a real Hilbert space and let $\left(\left\{S_{t}\right\},\left\{T_{t}\right\}\right)$ be a perturbation pair of $C_{0}$-semigroups acting on $G$. Let $\left\{G_{s, t}\right\},\left\{S_{t}^{0}\right\}$, and $\left\{T_{t}^{0}\right\}$ be as above. Then

(1) The system $\left(\left\{G_{a, b}\right\},\left\{S_{t}^{0}\right\}\right)$ forms a sum system.

(2) The pair of $C_{0}$-semigroups $\left(\left\{S_{t}^{0}\right\},\left\{T_{t}^{0}\right\}\right)$ is associated with $\left(\left\{G_{a, b}\right\},\left\{S_{t}^{0}\right\}\right)$. In consequence, the product system for the generalized CCR flow arising from $\left(\left\{S_{t}^{0}\right\},\left\{T_{t}^{0}\right\}\right)$ is isomorphic to the one arising from $\left(\left\{G_{a, b}\right\},\left\{S_{t}^{0}\right\}\right)$. 
(3) The product system for the generalized CCR flow arising from $\left(\left\{S_{t}\right\},\left\{T_{t}\right\}\right)$ is isomorphic to the product system arising from $\left(\left\{G_{a, b}\right\},\left\{S_{t}^{0}\right\}\right)$. In consequence, the generalized CCR flow arising from the pair $\left(\left\{S_{t}\right\},\left\{T_{t}\right\}\right)$ is cocycle conjugate to that arising from $\left(\left\{S_{t}^{0}\right\},\left\{T_{t}^{0}\right\}\right)$.

The following easy observation [8, Lemma 3.5] is very useful:

Lemma 2.10. Let $\left(\left\{S_{t}\right\},\left\{T_{t}\right\}\right)$ be a perturbation pair of $C_{0}$-semigroups acting on a real Hilbert space $G$. The two operators $S_{t} T_{t}^{*}$ and $I-S_{t} T_{t}^{*}$ are idempotents such that $\operatorname{Ran}\left(S_{t} T_{t}^{*}\right)=\operatorname{Ker}\left(I-S_{t} T_{t}^{*}\right)=\operatorname{Ran}\left(S_{t}\right)$ and $\operatorname{Ran}\left(I-S_{t} T_{t}^{*}\right)=\operatorname{Ker}\left(S_{t} T_{t}^{*}\right)=G_{0, t}$. In particular, the Hilbert space $G$ is a topological direct sum of $G_{0, t}$ and $\operatorname{Ran}\left(S_{t}\right)$.

\section{Divisibility}

Bhat and Srinivasan [5] introduced the notion of divisibility for sum systems. In this section we show that every sum system (in the sense of Definition 2.5) is divisible.

3.1. Addits. We first recall the definition of addits for a sum system, which were called additive units in [5].

Definition 3.1. Let $\left(\left\{G_{a, b}\right\},\left\{S_{t}\right\}\right)$ be a sum system. A real addit for the sum system $\left(\left\{G_{a, b}\right\},\left\{S_{t}\right\}\right)$ is a family $\left\{x_{t}\right\}_{t \in(0, \infty)}$ such that $x_{t} \in G_{0, t}$ for $\forall t \in(0, \infty)$, satisfying the following conditions:

(1) The map $t \mapsto\left\langle x_{t}, x\right\rangle$ is measurable for any $x \in G_{0, \infty}$.

(2) The cocycle relation $x_{s}+S_{s} x_{t}=x_{s+t}$, holds for $\forall s, t \in(0, \infty)$.

An imaginary addit for the sum system $\left(\left\{G_{a, b}\right\},\left\{S_{t}\right\}\right)$ is a family $\left\{y_{t}\right\}_{t \in(0, \infty)}$ such that $y_{t} \in G_{0, t}$ for $\forall t \in(0, \infty)$, satisfying the following conditions:

(1) The map $t \mapsto\left\langle y_{t}, y\right\rangle$ is measurable for $\forall y \in G_{0, \infty}$.

(2) The cocycle relation $\left(A_{s, t}^{*}\right)^{-1}\left(y_{s} \oplus\left(S_{s}^{*}\right)^{-1} y_{t}\right)=y_{s+t}$ holds for $\forall s, t, \in(0, \infty)$. We denote by $R \mathcal{A U}$ the set of all real addits and by $I \mathcal{A U}$ the set of all imaginary addits respectively, which are real linear spaces.

For a given real addit $\left\{x_{t}\right\}$, set $x_{s, t}=S_{s}\left(x_{t-s}\right) \in G_{s, t}$. Similarly for a given imaginary addit $\left\{y_{t}\right\}$, set $y_{s, t}=\left(S_{s}^{*}\right)^{-1}\left(y_{t-s}\right) \in G_{s, t}$. For $\left(s_{1}, s_{2}\right) \subset(0, s)$, we also set

$$
G_{0, s} \ni{ }^{s} y_{s_{1}, s_{2}}^{\prime}=\left(\tilde{A}^{*}\right)^{-1}\left(0 \oplus y_{s_{1}, s_{2}} \oplus 0\right)
$$

where $\tilde{A}$ is the map $G_{0, s_{1}} \oplus G_{s_{1}, s_{2}} \oplus G_{s_{2}, s} \ni x \oplus y \oplus z \mapsto x+y+z \in G_{0, s}$. By definition, we have ${ }^{s} y_{s_{1}, s_{2}}^{\prime} \in\left(G_{0, s_{1}} \vee G_{s_{2}, s}\right)^{\perp} \cap G_{0, s}$.

Definition 3.2. A sum system $\left(\left\{G_{a, b}\right\},\left\{S_{t}\right\}\right)$ is said to be divisible if each of $R \mathcal{A U}$ and $I \mathcal{A U}$ generates the sum system, that is,

$$
\begin{aligned}
G_{0, s} & =\overline{\operatorname{span}_{\mathbb{R}}\left\{x_{s_{1}, s_{2}} ;\left(s_{1}, s_{2}\right) \subseteq(0, s),\left\{x_{t}\right\} \in R \mathcal{A U}\right\}}, \\
G_{0, s} & =\overline{\operatorname{span}_{\mathbb{R}}\left\{{ }^{s} y_{s_{1}, s_{2}}^{\prime} ;\left(s_{1}, s_{2}\right) \subseteq(0, s),\left\{y_{t}\right\} \in I \mathcal{A U}\right\}} .
\end{aligned}
$$

Remark 3.3. Let $\left(\left\{H_{t}\right\},\left\{U_{s, t}\right\}\right)$ be a product system arising from $\left(\left\{G_{s, t}\right\},\left\{S_{t}\right\}\right)$. Bhat and Srinivasan [5] observed that every real addit $\left\{x_{t}\right\}$ gives a unitary operator $W\left(x_{t}\right)$ on $H_{t}$, and the family $\left\{W\left(x_{t}\right)\right\}_{t>0}$ forms an automorphism of the product system. For an imaginary addit $\left\{y_{t}\right\}$, the family $\left\{W\left(i y_{t}\right)\right\}_{t>0}$ forms an automorphism as well. Using this observation, they showed the following dichotomy result. 
Theorem 3.4 ([5]). Every product system arising from a divisible sum system is either type I or type III.

The following is [5, Proposition 37 (ii)], [8, Proposition 4.3].

Proposition 3.5. Let $\left(\left\{G_{(a, b)}\right\},\left\{S_{t}\right\}\right)$ be a sum system. If $\left\{x_{t}\right\} \in R \mathcal{A U}$ and $\left\{y_{t}\right\} \in$ $I \mathcal{A U}$, then

$$
\left\langle x_{t}, y_{t}\right\rangle=\left\langle x_{1}, y_{1}\right\rangle t, \forall t \in(0, \infty) .
$$

In general for any two intervals $\left(s_{1}, s_{2}\right),\left(t_{1}, t_{2}\right) \subset(0, s)$, it is true that

$$
\left\langle x_{s_{1}, s_{2}},{ }^{s} y_{t_{1}, t_{2}}^{\prime}\right\rangle=\left\langle x_{1}, y_{1}\right\rangle\left|\left(s_{1}, s_{2}\right) \cap\left(t_{1}, t_{2}\right)\right|,
$$

where |.| is the Lebesgue measure on $\mathbb{R}$.

3.2. Additive cocycles. Throughout this subsection, we assume that $G$ is a real Hilbert space, and that $\left(\left\{S_{t}\right\},\left\{T_{t}\right\}\right)$ is a perturbation pair of $C_{0}$-semigroups acting on $G$.

Definition 3.6. A real additive cocycle for the pair $\left(\left\{S_{t}\right\},\left\{T_{t}\right\}\right)$ is a family $\left\{c_{t}\right\}_{t \geq 0}$ such that $c_{t} \in \operatorname{Ker}\left(T_{t}^{*}\right)$ for $\forall t \in(0, \infty)$, satisfying the following conditions:

(1) The map $t \mapsto\left\langle c_{t}, x\right\rangle$ is measurable for any $x \in G$.

(2) The cocycle relation $c_{s}+S_{s} c_{t}=c_{s+t}$ holds for $\forall s, t \geq 0$.

An imaginary additive cocycle for the pair $\left(\left\{S_{t}\right\},\left\{T_{t}\right\}\right)$ is a family $\left\{d_{t}\right\}_{t \geq 0}$ such that $d_{t} \in \operatorname{Ker}\left(S_{t}^{*}\right)$ for $\forall t \in(0, \infty)$, satisfying the following conditions:

(1) The map $t \mapsto\left\langle y_{t}, y\right\rangle$ is measurable for any $y \in G$.

(2) The cocycle relation $d_{s}+T_{s} d_{t}=d_{s+t}$ holds for $\forall s, t \geq 0$.

Remark 3.7. Let $\left\{\alpha_{t}\right\}_{t \geq 0}$ be the generalized CCR flow associated with $\left(\left\{S_{t}\right\},\left\{T_{t}\right\}\right)$. Then every real additive cocycle $\left\{c_{t}\right\}_{t \geq 0}$ gives a family of unitary operators $\left\{W\left(c_{t}\right)\right\}_{t \geq 0}$ on $\Gamma\left(G^{\mathbb{C}}\right)$, which forms a gauge cocycle, that is, the unitary $W\left(x_{t}\right)$ is in the commutant of the image of $\alpha_{t}$ and the cocycle relation $W\left(x_{s+t}\right)=$ $W\left(x_{s}\right) \alpha_{t}\left(W\left(x_{t}\right)\right)$ holds for $s, t>0$. For an imaginary additive cocycle $\left\{d_{t}\right\}_{t \geq 0}$, the family $\left\{W\left(i d_{t}\right)\right\}_{t \geq 0}$ is a gauge cocycle as well.

In a similar way as in the proof of Proposition 3.5, we can show

Proposition 3.8. Let the notation be as above. If $\left\{c_{t}\right\}$ is a real additive cocycle and $\left\{d_{t}\right\}$ is an imaginary additive cocycle for $\left(\left\{S_{t}\right\},\left\{T_{t}\right\}\right)$, then

$$
\left\langle c_{t}, d_{t}\right\rangle=\left\langle c_{1}, d_{1}\right\rangle t, \forall t \in(0, \infty) .
$$

More generally, for any finite interval $(a, b) \subset(0, \infty)$, we set $c_{a, b}=S_{a} c_{b-a}$ and $d_{a, b}=T_{a} d_{b-a}$. Then

$$
\left\langle c_{s_{1}, s_{2}}, d_{t_{1}, t_{2}}\right\rangle=\left\langle c_{1}, d_{1}\right\rangle\left|\left(s_{1}, s_{2}\right) \cap\left(t_{1}, t_{2}\right)\right| .
$$

Clearly the real additive cocycles are the same as the real addits for the sum system $\left(\left\{G_{a, b}\right\},\left\{S_{t}^{0}\right\}\right)$ associated with the pair $\left(\left\{S_{t}\right\},\left\{T_{t}\right\}\right)$ as defined in subsection 2.6. We now describe a precise relationship between the imaginary additive cocycles for $\left(\left\{S_{t}\right\},\left\{T_{t}\right\}\right)$ and the imaginary addits for $\left(\left\{G_{a, b}\right\},\left\{S_{t}^{0}\right\}\right)$.

Let $A_{t}^{\prime}: G_{0, t} \oplus \operatorname{Ran}\left(S_{t}\right) \ni x \oplus y \mapsto x+y \in G$. Then it was shown in the proof of [8, Proposition 3.6] that $A_{t}^{\prime}$ is in $\mathcal{S}\left(G_{0, t} \oplus \operatorname{Ran}\left(S_{t}\right), G\right)$. The following lemma is essentially the same as [8, Remark 5.2]:

Lemma 3.9. Let the notation be as above. 
(1) If $\left\{y_{t}\right\}$ is an imaginary addit for the sum system $\left(\left\{G_{a, b}\right\},\left\{S_{t}^{0}\right\}\right)$ associated with the pair $\left(\left\{S_{t}\right\},\left\{T_{t}\right\}\right)$, then $\left\{\left(A_{t}^{\prime *}\right)^{-1}\left(y_{t} \oplus 0\right)\right\}$ is an imaginary additive cocycle for $\left(\left\{S_{t}\right\},\left\{T_{t}\right\}\right)$.

(2) If $\left\{d_{t}\right\}$ is an imaginary additive cocycle for $\left(\left\{S_{t}\right\},\left\{T_{t}\right\}\right)$, then $A_{t}^{\prime *} d_{t}$ is of the form $y_{t} \oplus 0$ and $\left\{y_{t}\right\}$ is an imaginary addit for the sum system $\left(\left\{G_{a, b}\right\},\left\{S_{t}^{0}\right\}\right)$.

(3) For every $x_{t}, y_{t} \in G_{0, t}$, we have $\left\langle x_{t},\left(A_{t}^{\prime *}\right)^{-1}\left(y_{t} \oplus 0\right)\right\rangle=\left\langle x_{t}, y_{t}\right\rangle$.

Proof. (1) Assume that $\left\{y_{t}\right\}$ is an imaginary addit for the sum system $\left(\left\{G_{a, b}\right\},\left\{S_{t}^{0}\right\}\right)$ and set $d_{t}=\left(A_{t}^{\prime *}\right)^{-1}\left(y_{t} \oplus 0\right)$. Then

$$
\left\langle d_{t}, S_{t} x\right\rangle=\left\langle y_{t} \oplus 0,0 \oplus S_{t} x\right\rangle=0, \forall x \in G,
$$

and hence $S_{t}^{*}\left(A_{t}^{\prime *}\right)^{-1}\left(y_{t} \oplus 0\right)=0$. Using $T_{s}=\left(A_{s}^{\prime *}\right)^{-1} A_{s}^{\prime-1}\left(S_{s}^{*}\right)^{-1}$ (see the proof of [8, Proposition 3.6,(c)]), we get

$$
\begin{aligned}
d_{s}+T_{s} d_{t} & =\left(A_{s}^{\prime *}\right)^{-1}\left(y_{s} \oplus 0\right)+\left(A_{s}^{\prime *}\right)^{-1} A_{s}^{\prime-1}\left(S_{s}^{*}\right)^{-1} d_{t} \\
& =\left(A_{s}^{\prime *}\right)^{-1}\left(y_{s} \oplus\left(S_{s}^{*}\right)^{-1}\left(A_{t}^{\prime *}\right)^{-1} y_{t}\right) .
\end{aligned}
$$

Since we have

$$
\begin{aligned}
A_{s}^{\prime}\left(I_{G_{0, s}} \oplus S_{s} A_{t}^{\prime}\right)\left(f \oplus g \oplus S_{t} h\right) & =f+S_{s} g+S_{s+t} h \\
& =A_{s+t}^{\prime}\left(A_{s, t}\left(I_{G_{0, s}} \oplus S_{s}\right) \oplus S_{s}\right)\left(f \oplus g \oplus S_{t} h\right),
\end{aligned}
$$

for $f \in G_{0, s}, g \in G_{0, t}$, and $h \in G$, we get $\left(A_{s}^{\prime *}\right)^{-1}\left(I_{G_{0, s}} \oplus\left(S_{s}{ }^{*}\right)^{-1}\left(A_{t}^{\prime *}\right)^{-1}\right)=\left(A_{s+t}^{\prime}{ }^{*}\right)^{-1}\left(\left(A_{s, t}{ }^{*}\right)^{-1}\left(I_{G_{0, s}} \oplus\left(S_{s}^{*}\right)^{-1}\right) \oplus\left(S_{s}{ }^{*}\right)^{-1}\right)$.

Therefore

$$
d_{s}+T_{s} d_{t}=\left(A_{s+t}^{\prime}{ }^{*}\right)^{-1}\left(\left(A_{s, t}{ }^{*}\right)^{-1}\left(y_{s} \oplus\left(S_{s}^{*}\right)^{-1} y_{t}\right) \oplus 0\right),
$$

and the cocycle relation for $\left\{y_{t}\right\}$ implies $d_{s}+T_{t} d_{t}=d_{s+t}$.

(2) Assume conversely that $\left\{d_{t}\right\}$ is an imaginary additive cocycle for $\left(\left\{S_{t}\right\},\left\{T_{t}\right\}\right)$. Then

$$
\left\langle A_{t}^{\prime *} d_{t}, 0 \oplus S_{t} x\right\rangle=\left\langle d_{t}, S_{t} x\right\rangle=0, \forall x \in G,
$$

and hence there exists $y_{t} \in G_{0, t}$ such that $A_{t}^{\prime *} d_{t}=y_{t} \oplus 0$. The above computation shows that $d_{s}+T_{t} d_{t}=d_{s+t}$ implies

$$
\left(A_{s, t}{ }^{*}\right)^{-1}\left(y_{s} \oplus\left(S_{s}^{*}\right)^{-1} y_{t}\right)=y_{s+t},
$$

and so $\left\{y_{t}\right\}$ is an imaginary addit for $\left(\left\{G_{a, b}\right\},\left\{S_{t}^{0}\right\}\right)$.

(3) follows from the definition of $A_{t}^{\prime}$.

Let $A$ and $B$ be the generators of the $C_{0}$-semigroups $\left\{S_{t}\right\}$ and $\left\{T_{t}\right\}$, respectively. In what follows, we often regard $G$ as a real subspace of its complexification $G^{\mathbb{C}}$ and identify operators on $G$ with their complex linear extensions to $G^{\mathbb{C}}$. Let

$$
C=\max \left\{\lim _{t \rightarrow+\infty} \frac{\log \left\|S_{t}\right\|}{t}, \lim _{t \rightarrow+\infty} \frac{\log \left\|T_{t}\right\|}{t}\right\},
$$

which is finite thanks to [14, page 232]. Then for $\operatorname{Re} z>C$, we have

$$
\begin{aligned}
& (z I-A)^{-1} f=\int_{0}^{\infty} e^{-t z} S_{t} f d t, \\
& (z I-B)^{-1} f=\int_{0}^{\infty} e^{-t z} T_{t} f d t .
\end{aligned}
$$


For a densely defined closed operator $R$ of $G$ with domain $D(R)$, we denote by $\mathcal{G}(R)$ the graph of $R$,

$$
\mathcal{G}(R)=\{f \oplus R f \in G \oplus G ; f \in D(R)\},
$$

by $\langle\cdot, \cdot\rangle_{R}$ the graph inner product,

$$
\langle f, g\rangle_{R}=\langle f, g\rangle+\langle R f, R g\rangle, \quad f, g \in D(R),
$$

and by $\|\cdot\|_{R}$ the graph norm $\|f\|=\langle f, f\rangle_{R}^{1 / 2}$. Then $\mathcal{G}(R)$ is a closed subspace of $G \oplus G$. Let $\mathcal{J}$ be the unitary of $G \oplus G$ defined by

$$
\mathcal{J}(f \oplus g)=g \oplus-f, \quad f, g \in G .
$$

Then $\mathcal{G}\left(R^{*}\right)=\mathcal{J G}(R)^{\perp}$.

The relation $T_{t}^{*} S_{t}=I$ implies $B \subset-A^{*}$ and $A \subset-B^{*}$. Let $\mathcal{K}$ be the orthogonal complement of $D(B)$ in $D\left(-A^{*}\right)$ with respect to the inner product $\langle\cdot, \cdot\rangle_{A^{*}}$. We always regard $\mathcal{K}$ as a Hilbert space equipped with the graph inner product. We let $\mathcal{K}^{\prime}$ be the orthogonal complement of $D(A)$ in $D\left(-B^{*}\right)$ with respect to the inner product $\langle\cdot, \cdot\rangle_{B^{*}}$ and regard it as a Hilbert space in a similar way.

Lemma 3.10. The restriction of $A^{*}$ to $\mathcal{K}$ is a unitary from $\mathcal{K}$ onto $\mathcal{K}^{\prime}$ and its inverse is the restriction of $B^{*}$ to $\mathcal{K}^{\prime}$.

Proof. By definition, we have

$$
\begin{aligned}
& \mathcal{G}\left(-A^{*}\right) \cap \mathcal{G}(B)^{\perp}=\left\{p \oplus-A^{*} p ; p \in \mathcal{K}\right\}, \\
& \mathcal{G}\left(-B^{*}\right) \cap \mathcal{G}(A)^{\perp}=\left\{q \oplus-B^{*} q ; q \in \mathcal{K}^{\prime}\right\} .
\end{aligned}
$$

On the other hand,

$$
\begin{aligned}
\mathcal{G}\left(-B^{*}\right) \cap \mathcal{G}(A)^{\perp} & =\mathcal{J}\left(\mathcal{G}(-B)^{\perp} \cap \mathcal{G}\left(A^{*}\right)\right) \\
& =\left\{A^{*} p \oplus-p ; p \in \mathcal{K}\right\},
\end{aligned}
$$

which shows that $\left.A^{*}\right|_{\mathcal{K}}$ is a linear isomorphism from $\mathcal{K}$ onto $\mathcal{K}^{\prime}$ whose inverse is $\left.B^{*}\right|_{\mathcal{K}^{\prime}}$. Since

$$
\left\langle A^{*} f, A^{*} g\right\rangle_{B^{*}}=\left\langle A^{*} f, A^{*} g\right\rangle+\left\langle B^{*} A^{*} f, B^{*} A^{*} g\right\rangle=\langle f, g\rangle+\left\langle A^{*} f, A^{*} g\right\rangle=\langle f, g\rangle_{A^{*}},
$$

for $f, g \in \mathcal{K}$, the restriction $\left.A^{*}\right|_{\mathcal{K}}$ is a unitary operator.

Definition 3.11. The index of a perturbation pair $\left(\left\{S_{t}\right\},\left\{T_{t}\right\}\right)$ of $C_{0}$-semigroups is the number $\operatorname{dim} \mathcal{K}=\operatorname{dim} \mathcal{K}^{\prime}$.

Recall $G_{0, t}=\operatorname{Ker} T_{t}^{*}$ and $G_{0, \infty}=\overline{\bigcup_{t>0} G_{0, t}}$. For $p \in \mathcal{K}$ and $t>0$, we set

$$
c(p)_{t}=\int_{0}^{t} S_{s} p d s+S_{t} A^{*} p-A^{*} p .
$$

Lemma 3.12. $\left\{c(p)_{t}\right\}_{t>0}$ is a real additive cocycle for $\left(\left\{S_{t}\right\},\left\{T_{t}\right\}\right)$.

Proof. It is easy to show the cocycle relation and all we have to show is $T_{t}^{*} c(p)_{t}=0$. Note that we have

$$
T_{t}^{*} c(p)_{t}=\int_{0}^{t} T_{t-s}^{*} p d s+A^{*} p-T_{t}^{*} A^{*} p .
$$


For $\operatorname{Re} z>2 C$,

$$
\begin{aligned}
\int_{0}^{\infty} e^{-t z} T_{t}^{*} c(p)_{t} d t & =\int_{0}^{\infty} e^{-t z} \int_{0}^{t} T_{t-s}^{*} p d s d t+\frac{1}{z} A^{*} p-\int_{0}^{\infty} e^{-t z} T_{t}^{*} A^{*} p d t \\
& =\int_{0}^{\infty} \int_{s}^{\infty} e^{-t z} T_{t-s}^{*} p d t d s+\frac{1}{z} A^{*} p-\left(z-B^{*}\right)^{-1} A^{*} p \\
& =\frac{1}{z}\left(z I-B^{*}\right)^{-1} p-\frac{1}{z}\left(z I-B^{*}\right)^{-1} B^{*} A^{*} p \\
& =0
\end{aligned}
$$

where we use Lemma 3.10. Since this holds for all Re $z>2 C$ and the map $t \mapsto$ $T_{t}^{*} c(p)_{t}$ is continuous, we conclude $T_{t}^{*} c(p)_{t}=0$.

Theorem 3.13. Let the notation be as above. Then

$$
\overline{\operatorname{span}\left\{S_{a} c(p)_{b} ; a+b \leq t, p \in \mathcal{K}\right\}}=G_{0, t}
$$

holds for all $t \in(0, \infty]$.

Proof. To prove the statement for $t=\infty$, it suffices to show

$$
\operatorname{span}\left\{S_{s} c(p)_{t} ; s, t>0, p \in \mathcal{K}\right\}^{\perp} \subset G_{0, \infty}{ }^{\perp} .
$$

First we claim that if $f \in \operatorname{span}\left\{c(p)_{t} ; t>0, p \in \mathcal{K}\right\}^{\perp}$, then $\left(z I-A^{*}\right)^{-1} f$ belongs to the domain of $B$ for every $\operatorname{Re} z>C$. Indeed,

$$
\begin{aligned}
0 & =\int_{0}^{\infty} e^{-t z}\left\langle f, c(p)_{t}\right\rangle d t \\
& =\int_{0}^{\infty} e^{-t z} \int_{0}^{t}\left\langle S_{s}^{*} f, p\right\rangle d s d t+\int_{0}^{\infty} e^{-t z}\left\langle S_{t}^{*} f, A^{*} p\right\rangle d t-\frac{1}{z}\left\langle f, A^{*} p\right\rangle \\
& =\frac{1}{z}\left\langle\left(z I-A^{*}\right)^{-1} f, p\right\rangle+\left\langle\left(z I-A^{*}\right)^{-1} f, A^{*} p\right\rangle-\frac{1}{z}\left\langle f, A^{*} p\right\rangle \\
& =\frac{1}{z}\left\langle\left(z I-A^{*}\right)^{-1} f, p\right\rangle+\frac{1}{z}\left\langle A^{*}\left(z I-A^{*}\right)^{-1} f, A^{*} p\right\rangle \\
& =\frac{\left\langle\left(z I-A^{*}\right)^{-1} f, p\right\rangle_{A^{*}}}{z} .
\end{aligned}
$$

This shows that $\left(z I-A^{*}\right)^{-1} f \in D\left(A^{*}\right)$ is in the orthogonal complement of $\mathcal{K}$ with respect to the inner product $\langle\cdot, \cdot\rangle_{A^{*}}$, which shows the claim.

Assume $f \in \operatorname{span}\left\{S_{s} c(p)_{t} ; s, t>0, p \in \mathcal{K}\right\}^{\perp}$. Then thanks to the above claim, we have $S_{t}^{*}\left(z I-A^{*}\right)^{-1} f=\left(z I-A^{*}\right)^{-1} S_{t}^{*} f \in D(B)$ for all $t \geq 0$ and $\operatorname{Re} z>C$. Let $g \in D\left(B^{*}\right)$. Then the function $t \mapsto\left\langle S_{t}^{*}\left(z I-A^{*}\right)^{-1} f, T_{t}^{*} g\right\rangle$ is differentiable and

$$
\begin{aligned}
\frac{d}{d t}\left\langle S_{t}^{*}\left(z I-A^{*}\right)^{-1} f, T_{t}^{*} g\right\rangle & =\left\langle A^{*} S_{t}^{*}\left(z I-A^{*}\right)^{-1} f, T_{t}^{*} g\right\rangle+\left\langle S_{t}^{*}\left(z I-A^{*}\right)^{-1} f, B^{*} T_{t}^{*} g\right\rangle \\
& =\left\langle A^{*} S_{t}^{*}\left(z I-A^{*}\right)^{-1} f, T_{t}^{*} g\right\rangle+\left\langle B S_{t}^{*}\left(z I-A^{*}\right)^{-1} f, T_{t}^{*} g\right\rangle \\
& =0,
\end{aligned}
$$

where we use $B \subset-A^{*}$. Therefore we get

$$
\left\langle T_{t} S_{t}^{*}\left(z I-A^{*}\right)^{-1} f, g\right\rangle=\left\langle\left(z I-A^{*}\right)^{-1} f, g\right\rangle
$$

for all $t \geq 0$ and $g \in D\left(B^{*}\right)$, and so $T_{t} S_{t}^{*}\left(z I-A^{*}\right)^{-1} f=\left(z I-A^{*}\right)^{-1} f$. Thanks to $G_{0, t}{ }^{\perp}=\operatorname{Ran}\left(T_{t}\right)$, we get $\left(z I-A^{*}\right)^{-1} f \in G_{0, \infty}{ }^{\perp}$ for all $\operatorname{Re} z>C$. Since $A^{*}$ is the 
generator of the $C_{0}$-semigroup $\left\{S_{t}^{*}\right\}_{t>0}$, we conclude

$$
f=\lim _{x \rightarrow+\infty} x\left(x I-A^{*}\right)^{-1} f \in G_{0, \infty}^{\perp} .
$$

Therefore the statement for $t=\infty$ is proven.

Recall from Lemma 2.10 that $G=G_{0, t}+\operatorname{Ran}\left(T_{t}\right)$ is a topological direct sum such that $I-S_{t} T_{t}^{*}$ is the projection onto the first component with respect to this direct sum decomposition. Let

$$
G_{a, b}^{0}=\operatorname{span}\left\{S_{s} c(p)_{t} ; a \leq s, s+t \leq b, p \in \mathcal{K}\right\} .
$$

Then on the one hand we have already seen that $G_{0, \infty}^{0}$ is dense in $G_{0, \infty}$, and on the other hand we have $\left(I-S_{t} T_{t}^{*}\right) G_{0, \infty}^{0}=G_{0, t}^{0}$. Therefore $G_{0, t}^{0}$ is dense in $G_{0, t}$.

By switching the roles of $\left\{S_{t}\right\}_{t>0}$ and $\left\{T_{t}\right\}_{t>0}$, we get the following:

Corollary 3.14. For $q \in \mathcal{K}^{\prime}$ and $t>0$, we set

$$
d(q)_{t}=\int_{0}^{t} T_{s} q d s+T_{t} B^{*} q-B^{*} q .
$$

Then $\left\{d(q)_{t}\right\}$ is an imaginary additive cocycle for $\left(\left\{S_{t}\right\},\left\{T_{t}\right\}\right)$ and

$$
\overline{\operatorname{span}\left\{T_{a} d(q)_{b} ; a+b \leq t, p \in \mathcal{K}\right\}}=\operatorname{Ker}\left(S_{t}^{*}\right)
$$

holds for all $t \in(0, \infty)$.

Theorem 3.15. Let the notation be as above. Then the following holds:

$$
\left\langle c(p)_{t}, d(q)_{t}\right\rangle=-t\left(\left\langle p, B^{*} q\right\rangle+\left\langle A^{*} p, q\right\rangle\right), \quad \forall p \in \mathcal{K}, \forall q \in \mathcal{K}^{\prime}
$$

Consequently, we have

$$
\left\langle c\left(p_{1}\right)_{t}, d\left(-A^{*} p_{2}\right)_{t}\right\rangle=t\left\langle p_{1}, p_{2}\right\rangle_{A^{*}}, \quad \forall p_{1}, p_{2} \in \mathcal{K},
$$

and the maps $\mathcal{K} \ni p \mapsto c(p)$ and $\mathcal{K}^{\prime} \ni q \mapsto d(q)$ are injective.

Proof. A direct computation shows

$$
\begin{aligned}
\left\langle c(p)_{t}, d(q)_{t}\right\rangle & =\int_{0}^{t}\left\langle c(p)_{t}, T_{s} q\right\rangle d s+\left\langle c(p)_{t}, T_{t} B^{*} q\right\rangle-\left\langle c(p)_{t}, B^{*} q\right\rangle \\
& =\int_{0}^{t}\left\langle c(p)_{s}+S_{s} c(p)_{t-s}, T_{s} q\right\rangle d s-\left\langle c(p)_{t}, B^{*} q\right\rangle \\
& =\int_{0}^{t}\left\langle c(p)_{t-s}, q\right\rangle d s-\left\langle c(p)_{t}, B^{*} q\right\rangle .
\end{aligned}
$$

Thus for $\operatorname{Re} z>2 C$,

$$
\begin{aligned}
\int_{0}^{\infty} e^{-t z}\left\langle c(p)_{t}, d(q)_{t}\right\rangle d t & =\int_{0}^{\infty} e^{-t z} \int_{0}^{t}\left\langle c(p)_{t-s}, q\right\rangle d s d t-\int_{0}^{\infty} e^{-t z}\left\langle c(p)_{t}, B^{*} q\right\rangle d t \\
& =\frac{1}{z} \int_{0}^{\infty} e^{-t z}\left\langle c(p)_{t}, q\right\rangle d t-\int_{0}^{\infty} e^{-t z}\left\langle c(p)_{t}, B^{*} q\right\rangle d t
\end{aligned}
$$


On the other hand, we have

$$
\begin{aligned}
\int_{0}^{\infty} e^{-t z} c(p)_{t} d t & =\int_{0}^{\infty} e^{-t z} \int_{0}^{t} S_{s} p d s d t+\int_{0}^{\infty} e^{-t z} S_{t} A^{*} p d t-\frac{1}{z} A^{*} p \\
& =\frac{1}{z}(z I-A)^{-1} p+(z I-A)^{-1} A^{*} p-\frac{1}{z} A^{*} p \\
& =\frac{1}{z}(z I-A)^{-1} p+\frac{1}{z} A(z I-A)^{-1} A^{*} p
\end{aligned}
$$

and so

$$
\begin{aligned}
z^{2} & \int_{0}^{\infty} e^{-t z}\left\langle c(p)_{t}, d(q)_{t}\right\rangle d t \\
& =\left\langle(z I-A)^{-1} p+A(z I-A)^{-1} A^{*} p, q\right\rangle-z\left\langle(z I-A)^{-1} p+A(z I-A)^{-1} A^{*} p, B^{*} q\right\rangle \\
& =\left\langle(z I-A)^{-1} p, A^{*} B^{*} q\right\rangle+\left\langle A(z I-A)^{-1} A^{*} p, q\right\rangle \\
& -z\left\langle(z I-A)^{-1} p, B^{*} q\right\rangle-z\left\langle(z I-A)^{-1} A^{*} p, A^{*} B^{*} q\right\rangle \\
& =\left\langle A(z I-A)^{-1} p, B^{*} q\right\rangle+\left\langle A(z I-A)^{-1} A^{*} p, q\right\rangle \\
& -z\left\langle(z I-A)^{-1} p, B^{*} q\right\rangle-z\left\langle(z I-A)^{-1} A^{*} p, q\right\rangle \\
& =-\left\langle p, B^{*} q\right\rangle-\left\langle A^{*} p, q\right\rangle .
\end{aligned}
$$

Therefore the inverse Laplace transformation implies the statement.

\subsection{Divisibility.}

Theorem 3.16. Every sum system is divisible.

Proof. Let $\left(\left\{G_{a, b},\left\{S_{t}\right\}\right\}\right)$ be a sum system and let $\left\{T_{t}\right\}$ be the $C_{0}$-semigroup constructed in subsection 2.5. For $\left\{S_{t}\right\}$ and $\left\{T_{t}\right\}$, we use the same notation as in the previous subsection. Theorem 3.13 implies

$$
G_{0, s}=\overline{\operatorname{span}\left\{x_{s_{1}, s_{2}} ;\left(s_{1}, s_{2}\right) \subseteq(0, s),\left\{x_{t}\right\} \in R \mathcal{A U}\right\}} .
$$

Lemma 3.9 and Corollary 3.14 imply

$$
G_{0, s} \cap\left\{{ }^{s} y_{a, b}^{\prime} ; y \in I \mathcal{A U}, 0<a, b<s\right\}^{\perp} \subset \operatorname{Ker}\left(T_{s}^{*}\right) \cap \operatorname{Ker}\left(S_{s}^{*}\right)^{\perp} .
$$

Since $S_{s}$ has a left inverse $T_{s}^{*}$, the range of $S_{s}$ is closed, and we have $\operatorname{Ker}\left(S_{s}^{*}\right)^{\perp}=$ $\operatorname{Ran}\left(S_{s}\right)$. Therefore to prove

$$
G_{0, s}=\overline{\operatorname{span}_{\mathbb{R}}\left\{{ }^{s} y_{s_{1}, s_{2}}^{\prime} ;\left(s_{1}, s_{2}\right) \subseteq(0, s),\left\{y_{t}\right\} \in I \mathcal{A U}\right\}},
$$

it suffices to show $\operatorname{Ker}\left(T_{t}^{*}\right) \cap \operatorname{Ran}\left(S_{t}\right)=\{0\}$. Indeed, let $f \in \operatorname{Ker}\left(T_{t}^{*}\right) \cap \operatorname{Ran}\left(S_{t}\right)$. Then there exists $g \in G$ such that $f=S_{t} g$, and $T_{t}^{*} f=0$ on the other hand. Therefore $0=T_{t}^{*} S_{t} g=g$ and $f=0$. This proves the statement.

The above theorem together with Theorem 3.4 implies

Corollary 3.17. Every product system arising from a sum system is either of type I or type III. Every generalized CCR flow is either of type I or type III.

In the rest of this section, we keep employing the same assumption and notation as in the proof of Theorem 3.16 and the previous subsection.

For $x=\left\{x_{t}\right\} \in R \mathcal{A U}$ and $y=\left\{y_{t}\right\} \in I A U$, we set $b_{G}(x, y)=\left\langle x_{1}, y_{1}\right\rangle$. Then Proposition 3.5. Theorem 3.15] and Theorem 3.16 show that $b_{G}$ is non-degenerate as a bilinear form $b_{G}: R \mathcal{A U} \times I \mathcal{A U} \rightarrow \mathbb{R}$. 
Lemma 3.18. Let the notation be as above. Then

$$
\operatorname{dim} \mathcal{K}=\operatorname{dim} \mathcal{K}^{\prime}=\operatorname{dim} R \mathcal{A U}=\operatorname{dim} I \mathcal{A U} .
$$

Proof. We already know from Lemma 3.10, Theorem 3.15, and the non-degeneracy of $b_{G}$ that

$$
\operatorname{dim} \mathcal{K}=\operatorname{dim} \mathcal{K}^{\prime} \leq \operatorname{dim} R \mathcal{A U}=\operatorname{dim} I \mathcal{A U}
$$

holds. Assume that $\operatorname{dim} \mathcal{K}$ is finite. If $\operatorname{dim} I \mathcal{A U}$ were strictly larger than $\operatorname{dim} \mathcal{K}$, there would exist $\left\{y_{t}\right\} \in I \mathcal{A U} \backslash\{0\}$ such that $b_{G}(c(p), y)=0$ for all $p \in \mathcal{K}$. However, this contradicts Proposition 3.5 and Theorem 3.13. Thus we get the statement.

The above lemma allows us to introduce the index of a sum system.

Definition 3.19. For a sum system $\left(\left\{G_{a, b}\right\},\left\{S_{t}\right\}\right)$, the index ind $G$ is the number

$$
\operatorname{dim} \mathcal{K}=\operatorname{dim} \mathcal{K}^{\prime}=\operatorname{dim} R \mathcal{A U}=\operatorname{dim} I \mathcal{A U} .
$$

Now we discuss an appropriate topology of $R \mathcal{A U}$ and $I \mathcal{A U}$. For $p \in \mathcal{K}$, we set $x(p)_{t}=c(p)_{t}$ and $y(p)_{t} \oplus 0=\left(A_{t}^{\prime}\right)^{*} d\left(-A^{*} p\right)_{t}$. Then $x(p) \in R \mathcal{A U}, y(p) \in I \mathcal{A} \mathcal{U}$, and $b_{G}\left(x\left(p_{1}\right), y\left(p_{2}\right)\right)=\left\langle p_{1}, p_{2}\right\rangle_{A^{*}}$ thanks to Lemma 3.9 and Theorem 3.15 .

Lemma 3.20. For the linear space $\{x(p) \in R \mathcal{A U} ; p \in \mathcal{K}\}$, the following three topologies coincide:

(1) the topology of uniform convergence on every compact subset of $[0, \infty)$,

(2) the topology given by the metric $d_{1}\left(\left(x\left(p_{1}\right), x\left(p_{2}\right)\right)=\left\|x\left(p_{1}\right)_{1}-x\left(p_{2}\right)_{1}\right\|\right.$,

(3) the topology given by the metric $d_{2}\left(x\left(p_{1}\right), x\left(p_{2}\right)\right)=\left\|p_{1}-p_{2}\right\|_{A^{*}}$.

For the linear space $\{y(p) \in I \mathcal{A U} ; p \in \mathcal{K}\}$, the following three topologies coincide:

(1) the topology of uniform convergence on every compact subset of $[0, \infty)$,

(2) the topology given by the metric $d_{1}\left(\left(y\left(p_{1}\right), y\left(p_{2}\right)\right)=\left\|y\left(p_{1}\right)_{1}-y\left(p_{2}\right)_{1}\right\|\right.$,

(3) the topology given by the metric $d_{2}\left(y\left(p_{1}\right), y\left(p_{2}\right)\right)=\left\|p_{1}-p_{2}\right\|_{A^{*}}$.

Proof. It is easy to show that there exists a positive constant $C_{t}$, increasing in $t>0$, such that

$$
\begin{aligned}
\left\|x(p)_{t}\right\| \leq C_{t}\|p\|_{A^{*}}, \quad \forall p \in \mathcal{K}, \\
\left\|y(p)_{t}\right\| \leq C_{t}\|p\|_{A^{*}}, \quad \forall p \in \mathcal{K} .
\end{aligned}
$$

Since

$$
t\|p\|_{A^{*}}^{2}=\left\langle x(p)_{t}, y(p)_{t}\right\rangle \leq\left\|x(p)_{t}\right\|\left\|y(p)_{t}\right\| \leq C_{t}\|p\|_{A^{*}}\left\|x(p)_{t}\right\|,
$$

we get

$$
\frac{t}{C_{t}}\|p\|_{A^{*}} \leq\left\|x(p)_{t}\right\| \leq C_{t}\|p\|_{A^{*}},
$$

and in the same way,

$$
\frac{t}{C_{t}}\|p\|_{A^{*}} \leq\left\|y(p)_{t}\right\| \leq C_{t}\|p\|_{A^{*}}
$$

This proves the statement.

Remark 3.21. The results obtained in this section substantially simplify arguments in [8, Section 5] for addits in concrete examples, although the author could not find the explicit formula (3.1) without making the concrete computation in 8, Section $5]$. 


\section{TYPE III CRITERION}

A type III criterion was obtained in 8 for a product system arising from a sum system of finite index. Now the description of additive cocycles in the previous section allows us to show the criterion in full generality.

Lemma 4.1. Let $G$ be a real Hilbert space and let $(w, K)$ be a continuous unitary representation of $G$ (as an additive group) on a complex Hilbert space $K$ without containing the trivial representation. Let $c: G \rightarrow K$ be a continuous 1-cocycle; that is, the map $c$ satisfies the cocycle relation

$$
c(r+s)=c(r)+w(r) c(s), \quad \forall r, s \in G .
$$

Then the following equation holds for all $r, s \in G$ :

$$
\langle c(r), w(r) c(s)\rangle=\langle c(s), w(s) c(r)\rangle .
$$

Proof. Let $r, s \in G$ and let $G_{0}=\operatorname{span}\{r, s\}$. Let

$$
K_{0}=\left\{f \in K ; w(g) f=f, \forall g \in G_{0}\right\}
$$

and let $K_{1}=K_{0}^{\perp}$. Then $K_{0}$ and $K_{1}$ are invariant under the unitary representation $w$. Let $c_{0}(g)$ and $c_{1}(g)$ be the projections of $c(g)$ onto $K_{0}$ and $K_{1}$, respectively. Then $c_{0}$ and $c_{1}$ are cocycles. Thanks to the cocycle relation, for every $g \in G$ and $h \in G_{0}$, we have

$$
c_{0}(g)+w(g) c_{0}(h)=c_{0}(g+h)=c_{0}(h)+w(h) c_{0}(g)=c_{0}(h)+c_{0}(g),
$$

and we get $w(g) c_{0}(h)=c_{0}(h)$. Since $w$ does not contain the trivial representation, we have $c_{0}(h)=0$ for all $h \in G_{0}$. Now we can apply [8, Lemma 4.7] to $c_{1}$.

Let $K$ be a complex Hilbert space. Recall that the automorphism group $G_{K}$ of the exponential product system of index $\operatorname{dim} K$ is described as follows (see [2], 4]): Let $U(K)$ be the unitary group of $K$. Then $G_{K}$ is homeomorphic to $\mathbb{R} \times K \times U(K)$ with the group operation

$$
(a, \xi, u) \cdot(b, \eta, v)=(a+b+\operatorname{Im}\langle\xi, u \eta\rangle, \xi+u \eta, u v) .
$$

For $(a, \xi, u) \in G_{K}$, the corresponding automorphism is realized by the family of unitary operators $e^{i a t} W\left(1_{(0, t]} \xi\right) \operatorname{Exp}\left(1_{(0, t]} u\right), t>0$. The following is 8 , Lemma 4.8].

Lemma 4.2. Let $G$ be an abelian group and let $\rho: G \ni r \mapsto(a(r), \xi(r), u(r)) \in G_{K}$ be a map. Then $\rho$ is a homomorphism if and only if the following relation holds for every $r, s \in G$ :

$$
\begin{gathered}
a(r+s)=a(r)+a(s)+\operatorname{Im}\langle\xi(r), u(r) \xi(s)\rangle, \\
\xi(r+s)=\xi(r)+u(r) \xi(s), \\
u(r+s)=u(r) u(s) .
\end{gathered}
$$

In particular, when $u(r)=1$ for all $r \in G$, then $\rho$ is a homomorphism if and only if

$$
\begin{gathered}
a(r+s)=a(r)+a(s), \\
\xi(r+s)=\xi(r)+\xi(s), \\
\operatorname{Im}\langle\xi(r), \xi(s)\rangle=0 .
\end{gathered}
$$


For a sum system $\left(\left\{G_{a, b}\right\},\left\{S_{t}\right\}\right)$, we denote by $\left\{T_{t}\right\}$ the $C_{0}$-semigroup constructed in subsection 2.5. We use the same notation as in the previous section such as $x(p), y(p)$, and $\mathcal{K}$. Let

$$
\begin{aligned}
G_{0, t}^{0} & =\operatorname{span}\left\{x(p)_{s_{1}, s_{2}} ;\left(s_{1}, s_{2}\right) \subseteq(0, t), p \in \mathcal{K}\right\} \subseteq G_{0, t}, \\
G_{0, t}^{0}{ }^{\prime} & =\operatorname{span}\left\{{ }^{t} y(p)_{s_{1}, s_{2}}^{\prime} ;\left(s_{1}, s_{2}\right) \subseteq(0, t), p \in \mathcal{K}\right\} \subseteq G_{0, t} .
\end{aligned}
$$

Then they are dense in $G_{0, t}$. For a subset $X \subset(0, \infty)$, we denote by $1_{X}$ the characteristic function of $X$.

Theorem 4.3. Let $\left(\left\{G_{a, b}\right\},\left\{S_{t}\right\}\right)$ be a sum system. Then the following conditions are equivalent.

(1) The product system $\left(H_{t}, U_{s, t}\right)$ arising from $\left(\left\{G_{a, b}\right\},\left\{S_{t}\right\}\right)$ is of type $I$.

(2) The sum system $\left(\left\{G_{a, b}\right\},\left\{S_{t}\right\}\right)$ is isomorphic to the shift sum system of index ind $G$. More precisely, there exists an invertible operator $F$ from $\mathcal{K}$ onto a real Hilbert space $K_{\mathbb{R}}$ such that the map

$$
\begin{array}{r}
G_{0, t} \ni x(p)_{a, b} \mapsto 1_{(a, b]} \otimes F p \in L^{2}(0, t)_{\mathbb{R}} \otimes K_{\mathbb{R}}, \\
(a, b) \subset(0, t), p \in \mathcal{K},
\end{array}
$$

extends to an isomorphism from the sum system $\left(\left\{G_{a, b}\right\},\left\{S_{t}\right\}\right)$ to the sum system $\left(\left\{L^{2}\left((a, b), K_{\mathbb{R}}\right)\right\},\left\{S_{t}^{\prime}\right\}\right)$, where $\left\{S_{t}^{\prime}\right\}$ is the shift semigroup of $L^{2}\left((0, \infty), K_{\mathbb{R}}\right)$.

Proof. The implication from (2) to (1) is trivial.

Assume that $\left(\left\{G_{a, b}\right\},\left\{S_{t}\right\}\right)$ is of type I. Then it is isomorphic to an exponential product system (see [2], 4]). For $t>0$, let

$$
V_{t}: \Gamma\left(G_{0, t}\right) \rightarrow \Gamma\left(L^{2}((0, t), K)\right)
$$

be a family of unitary operators, implementing the isomorphism between the above product systems. Here $K$ is some separable complex Hilbert space.

For each $p \in \mathcal{K}$, the families $\left\{W\left(x(p)_{t}\right)\right\}_{t>0}$ and $\left\{W\left(i y(p)_{t}\right)\right\}_{t>0}$ form automorphisms for the product system $\left(H_{t}, U_{s, t}\right)$ (see [5, Theorem 26]) satisfying the relations:

$$
\begin{gathered}
W\left(x\left(p_{1}\right)_{t}\right) W\left(x\left(p_{2}\right)_{t}\right)=W\left(x\left(p_{1}+p_{2}\right)_{t}\right), \quad \forall p_{1}, p_{2} \in \mathcal{K}, \\
W\left(i y\left(p_{1}\right)_{t}\right) W\left(i y\left(p_{2}\right)_{t}\right)=W\left(i y\left(p_{1}+p_{2}\right)_{t}\right), \quad \forall p_{1}, p_{2} \in \mathcal{K}, \\
W\left(x\left(p_{1}\right)_{t}\right) W\left(i y\left(p_{2}\right)_{t}\right)=e^{-2 i t\left\langle p_{1}, p_{2}\right\rangle} W\left(i y\left(p_{2}\right)_{t}\right) W\left(x\left(p_{1}\right)_{t}\right), \quad \forall p_{1}, p_{2} \in \mathcal{K} .
\end{gathered}
$$

In the last equation we use $\left\langle x\left(p_{1}\right)_{t}, y\left(p_{2}\right)_{t}\right\rangle=t\left\langle p_{1}, p_{2}\right\rangle_{A^{*}}$. Therefore, thanks to Lemma 3.20 , there exist two continuous homomorphisms

$$
\begin{gathered}
\rho: \mathcal{K} \ni p \mapsto(a(p), \xi(p), u(p)) \in G_{K}, \\
\sigma: \mathcal{K} \ni p \mapsto(b(p), \eta(p), v(p)) \in G_{K},
\end{gathered}
$$

satisfying

$$
\begin{aligned}
V_{t} W\left(x(p)_{t}\right) V_{t}^{*}=e^{i t a(p)} W\left(1_{(0, t]} \xi(p)\right) \operatorname{Exp}\left(1_{(0, t]} u(p)\right), & \forall p \in \mathcal{K}, \\
V_{t} W\left(i y(p)_{t}\right) V_{t}^{*}=e^{i t b(p)} W\left(1_{(0, t]} \eta(p)\right) \operatorname{Exp}\left(1_{(0, t]} v(p)\right), & \forall p \in \mathcal{K} .
\end{aligned}
$$


Equation (4.9) implies that in addition to the relations in Lemma 4.2, we have

$$
\begin{gathered}
2\left\langle p_{1}, p_{2}\right\rangle_{A^{*}}=\operatorname{Im}\left\langle\eta\left(p_{2}\right), v\left(p_{2}\right) \xi\left(p_{1}\right)\right\rangle-\operatorname{Im}\left\langle\xi\left(p_{1}\right), u\left(p_{1}\right) \eta\left(p_{2}\right)\right\rangle, \\
\xi\left(p_{1}\right)+u\left(p_{1}\right) \eta\left(p_{2}\right)=\eta\left(p_{2}\right)+v\left(p_{2}\right) \xi\left(p_{1}\right), \\
u\left(p_{1}\right) v\left(p_{2}\right)=v\left(p_{2}\right) u\left(p_{1}\right) .
\end{gathered}
$$

Let $w\left(\left(p_{1}, p_{2}\right)\right)=u\left(p_{1}\right) v\left(p_{2}\right)$ and $c\left(\left(p_{1}, p_{2}\right)\right)=\xi\left(p_{1}\right)+u\left(p_{1}\right) \eta\left(p_{2}\right)$. Then (4.3) and (4.14) imply that $(K, w)$ is a continuous unitary representation of $\mathcal{K}^{2}$, and (4.2) and (4.13) imply that $c$ is a continuous 1-cocycle. Let

$$
K_{0}=\left\{z \in K ; w(g) z=z, \forall g \in \mathcal{K}^{2}\right\},
$$

and let $K_{1}=K_{0}^{\perp}$. Let $\xi_{i}(p)$ be the projection of $\xi(p)$ to $K_{i}$ and let $\eta_{i}(p)$ be the projection of $\eta(p)$ to $K_{i}$. Then Lemma 4.1 implies

$$
\left\langle\xi_{1}\left(p_{1}\right), u\left(p_{1}\right) \eta_{1}\left(p_{2}\right)\right\rangle=\left\langle\eta_{1}\left(p_{2}\right), v\left(p_{2}\right) \xi_{1}\left(p_{1}\right)\right\rangle,
$$

and Equation (4.12) is equivalent to

$$
\left\langle p_{1}, p_{2}\right\rangle_{A^{*}}=\operatorname{Im}\left\langle\eta_{0}\left(p_{2}\right), \xi_{0}\left(p_{1}\right)\right\rangle .
$$

We claim that $u$ and $v$ are trivial, that is, $K_{1}=\{0\}$. Assume that $K_{1}$ is not trivial. Let $0<r<s<t$. Then it is routine work to show

$$
\begin{gathered}
V_{t} W\left(x(p)_{r, s}\right) V_{t}^{*}=e^{i(s-r) a(p)} W\left(1_{(r, s]} \xi(p)\right) \operatorname{Exp}\left(1_{(0, r]}+1_{(r, s]} u(p)+1_{(s, t]}\right), \\
V_{t} W\left(i^{t} y(p)_{r, s}^{\prime}\right) V_{t}^{*}=e^{i(s-r) b(p)} W\left(1_{(r, s]} \eta(p)\right) \operatorname{Exp}\left(1_{(0, r]}+1_{(r, s]} v(p)+1_{(s, t]}\right) .
\end{gathered}
$$

By definition of $K_{1}$, there exists $p_{0} \in \mathcal{K}$ such that either $u\left(p_{0}\right)$ or $v\left(p_{0}\right)$ is not trivial. Thus we assume that $u\left(p_{0}\right) \neq 1$ (the case with non-trivial $v\left(p_{0}\right)$ can be treated in the same way). Direct computation using Lemma 4.1 and (4.1) shows that the operator $W\left(1_{(0, t]} \xi_{1}\left(p_{0}\right)\right) \operatorname{Exp}\left(1_{(0, t]} u\left(p_{0}\right)\right)$ commutes with $V_{t} W\left(x(p)_{r, s}\right) V_{t}^{*}$ and $V_{t} W\left(i^{t} y(p)_{r, s}^{\prime}\right) V_{t}^{*}$ for all $p \in \mathcal{K}$ and $0<r<s<t$. However, this contradicts the irreducibility of the vacuum representation of the Weyl algebra, since the sets $\left\{x(p)_{r, s} ;(r, s) \subseteq(0, t), p \in \mathcal{K}\right\}$ and $\left\{{ }^{t} y(p)_{r, s}^{\prime} ;(r, s) \subseteq(0, t), p \in \mathcal{K}\right\}$ are total in $G_{0, t}$. Hence $K=K_{0}$.

Next we claim that $\xi(\mathcal{K})+\eta(\mathcal{K})$ is dense in $K$. If this were not the case, there would exist a non-zero $\zeta \in K$ orthogonal to $\xi(\mathcal{K})$ and $\eta(\mathcal{K})$ with respect to the real inner product $\operatorname{Re}\langle\cdot, \cdot\rangle$. Again we can show that $W\left(i 1_{(0, t]} \zeta\right)$ would commute with $V_{t} W\left(x(p)_{r, s}\right) V_{t}^{*}$ and $V_{t} W\left(i^{t} y(p)_{r, s}^{\prime}\right) V_{t}^{*}$, for all $p \in \mathcal{K}$ and $0<r<s<t$, which is a contradiction. Therefore we conclude that $\xi(\mathcal{K})+\eta(\mathcal{K})$ is dense in $K$.

In the above argument, we have shown the following: there exist continuous homomorphisms (a priori as additive groups) $\xi: \mathcal{K} \rightarrow K, \eta: \mathcal{K} \rightarrow K, a: \mathcal{K} \rightarrow \mathbb{R}$, and $b: \mathcal{K} \rightarrow \mathbb{R}$ satisfying

$$
\begin{gathered}
V_{t} W\left(x(p)_{t}\right) V_{t}^{*}=e^{i t a(p)} W\left(1_{(0, t]} \xi(p)\right), \\
V_{t} W\left(i y(p)_{t}\right) V_{t}^{*}=e^{i t b(p)} W\left(1_{(0, t]} \eta(p)\right), \\
\operatorname{Im}\left\langle\xi\left(p_{1}\right), \xi\left(p_{2}\right)\right\rangle=0, \quad \forall p_{1}, p_{2} \in \mathcal{K}, \\
\operatorname{Im}\left\langle\eta\left(y\left(p_{1}\right)\right), \eta\left(y\left(p_{2}\right)\right)\right\rangle=0, \quad \forall p_{1}, p_{2} \in \mathcal{K}, \\
\left\langle p_{1}, p_{2}\right\rangle_{A^{*}}=\operatorname{Im}\left\langle\eta\left(p_{2}\right), \xi\left(p_{1}\right)\right\rangle, \quad \forall p_{1}, p_{2} \in \mathcal{K} .
\end{gathered}
$$

Since $\xi, \eta, a$, and $b$ are continuous, these maps are in fact linear. 
We can get rid of $a$ and $b$ in the above. Since $a$ and $b$ are bounded linear functionals, there exist $a_{0}, b_{0} \in \mathcal{K}$ such that $a(p)=\left\langle p, a_{0}\right\rangle_{A^{*}}$ and $b(p)=\left\langle p, b_{0}\right\rangle_{A^{*}}$ for all $p \in \mathcal{K}$. Let

$$
\zeta=\frac{1}{2} \xi\left(b_{0}\right)-\frac{1}{2} \eta\left(a_{0}\right) .
$$

Then a direct computation yields $\operatorname{Im}\langle\zeta, \xi(p)\rangle=-a(p) / 2$ and $\operatorname{Im}\langle\zeta, \eta(p)\rangle=$ $-b(p) / 2$ for all $p \in \mathcal{K}$. By replacing $V_{t}$ with $W\left(1_{(0, t]} \zeta\right) V_{t}$, we may and do assume $a$ and $b$ are trivial.

Equation (4.21) shows that $\xi$ and $\eta$ are injective and

$$
\|p\|_{A^{*}}^{2}=\operatorname{Im}\langle\eta(p), \xi(p)\rangle \leq\|\eta(p)\|\|\xi(p)\| \leq\|\eta\|\|p\|_{A^{*}}\|\xi(p)\|,
$$

which implies $\|p\|_{A^{*}} \leq\|\eta\|\|\xi(p)\|$. In the same way we get $\|p\|_{A^{*}} \leq\|\xi\|\|\eta(p)\|$. In particular, the maps $\xi$ and $\eta$ are invertible from $\mathcal{K}$ onto their images, which are closed. Thanks to Equation (4.19), the subspace $\xi(\mathcal{K})+i \xi(\mathcal{K})$ is a closed complex subspace of $K$. We claim $K=\xi(\mathcal{K})+i \xi(\mathcal{K})$. Assume $\zeta \in(\xi(\mathcal{K})+i \xi(\mathcal{K}))^{\perp}$. Then since $\xi(\mathcal{K})+\eta(\mathcal{K})$ is dense in $\mathcal{K}$, there exist two sequences $\left\{p_{n}\right\}$ and $\left\{q_{n}\right\}$ in $\mathcal{K}$ such that $\left\{\xi\left(p_{n}\right)+\eta\left(q_{n}\right)\right\}$ converges to $\zeta$. For $p \in \mathcal{K}$, we have

$$
\lim _{n \rightarrow \infty}\left\langle\xi\left(p_{n}\right)+\eta\left(q_{n}\right), \xi(p)\right\rangle=0, \quad \forall p \in \mathcal{K} .
$$

This implies

$$
\begin{aligned}
& 0=\lim _{n \rightarrow \infty} \operatorname{Im}\left\langle\xi\left(p_{n}\right)+\eta\left(q_{n}\right), \xi(p)\right\rangle=\lim _{n \rightarrow \infty}\left\langle q_{n}, p\right\rangle, \\
& 0=\lim _{n \rightarrow \infty} \operatorname{Re}\left\langle\xi\left(p_{n}\right)+\eta\left(q_{n}\right), \xi(p)\right\rangle=\lim _{n \rightarrow \infty}\left(\left\langle\xi\left(p_{n}\right), \xi(p)\right\rangle+\operatorname{Re}\left\langle\eta\left(q_{n}\right), \xi(p)\right\rangle\right) .
\end{aligned}
$$

The first equation means that the sequence $\left\{q_{n}\right\}$ converges to 0 weakly, and as a consequence $\left\{\eta\left(q_{n}\right)\right\}$ converges to 0 weakly. Thus the second equation implies that the sequence $\left\{\xi\left(p_{n}\right)\right\}$ converges to 0 weakly as well, and so $\zeta=0$. This shows that $\xi(\mathcal{K})+i \xi(\mathcal{K})=K$ and we may identify $K$ with the complexification of $\xi(\mathcal{K})$. We denote $\xi(\mathcal{K})$ by $K_{\mathbb{R}}$.

From now on, we regard $\xi$ as an invertible operator from $\mathcal{K}$ onto the real Hilbert space $K_{\mathbb{R}}$. We claim that there exists a self-adjoint operator $L \in \mathbb{B}\left(K_{\mathbb{R}}\right)$, eventually shown to be 0 , such that $\eta \xi^{*}(f)=L f+i f$ for all $f \in K_{\mathbb{R}}$. Since $K$ is the complexification of $K_{\mathbb{R}}$, there exist two operators $L, L^{\prime} \in \mathbb{B}\left(K_{\mathbb{R}}\right)$ such that $\eta \xi^{*} f=$ $L f+i L^{\prime} f$. For $f, g \in K_{\mathbb{R}}$, we have

$$
\operatorname{Im}\left\langle\eta \xi^{*} f, g\right\rangle=\operatorname{Im}\left\langle\eta \xi^{*} f, \xi \xi^{-1} g\right\rangle=\left\langle\xi^{*} f, \xi^{-1} g\right\rangle=\langle f, g\rangle,
$$

which shows $L^{\prime}=1$. We also have

$$
0=\operatorname{Im}\left\langle\eta \xi^{*} f, \eta \xi^{*} g\right\rangle=\langle f, L g\rangle-\langle L f, g\rangle,
$$

which shows that $L$ is self-adjoint.

Since $G_{0, t}^{0}+i G_{0, t}^{0^{\prime}}$ is dense in $G_{0, t}^{\mathbb{C}}$, Lemma 2.4 implies that there exists an equivalence operator $R \in \mathcal{S}\left(G_{0, t}^{\mathbb{C}}, L^{2}((0, t), K)\right)$, where $G_{0, t}^{\mathbb{C}}$ and $L^{2}((0, t), K)$ are regarded as real Hilbert spaces with real inner product $\operatorname{Re}\langle\cdot, \cdot\rangle$, such that for every $(r, s) \subset(0, t)$ and $p \in \mathcal{K}$,

$$
\begin{gathered}
R x(p)_{r, s}=1_{(r, s]} \xi(p), \\
R i^{t} y(p)_{r, s}^{\prime}=1_{(r, s]} \eta(p) .
\end{gathered}
$$

We first claim that $L$ is a compact operator. Assume this is not the case. Then there would exist a non-zero real number $\lambda$ and an orthonormal system $\left\{f_{n}\right\}$ in $K_{\mathbb{R}}$ such that $\left\{\left\|L f_{n}-\lambda f_{n}\right\|\right\}$ converges to zero. We set $p_{n}=\xi^{-1} f_{n}$ and $q_{n}=\xi^{*} f_{n}$. 
Then $\left\{p_{n}\right\}$ and $\left\{q_{n}\right\}$ converge to 0 weakly. Since $\operatorname{Re}\left\langle x\left(p_{n}\right)_{t}, i y\left(q_{n}\right)_{t}\right\rangle=0$ and $R$ is an equivalence operator, we have

$$
\lim _{n \rightarrow \infty} \operatorname{Re}\left\langle R x\left(p_{n}\right)_{t}, \operatorname{Riy}\left(q_{n}\right)_{t}\right\rangle=0 .
$$

On the other hand, we have

$$
\operatorname{Re}\left\langle\operatorname{Rx}\left(p_{n}\right)_{t}, \operatorname{Riy}\left(q_{n}\right)_{t}\right\rangle=t \operatorname{Re}\left\langle\xi\left(p_{n}\right), \eta\left(q_{n}\right)\right\rangle=t \operatorname{Re}\left\langle e_{n}, L e_{n}\right\rangle \rightarrow t \lambda \quad(n \rightarrow \infty),
$$

which is a contradiction.

Now we show that $L=0$. Assume on the contrary that $L \neq 0$. Since $L$ is a compact self-adjoint operator, there would exist a non-zero real eigenvalue $\lambda$ with normalized eigenvector $f$. We set $p=\xi^{-1} f$ and $q=\xi^{*} f$. Let

$$
\begin{aligned}
& \tilde{x}_{n}=\sqrt{2^{n}} x(p)_{2^{-n}}, \\
& \tilde{y}_{n}=\sqrt{2^{n} t} y(q)_{2^{-n}}^{\prime} .
\end{aligned}
$$

Since $\left\{R \tilde{x}_{n}\right\}$ and $\left\{R i \tilde{y}_{n}\right\}$ are bounded, the two sequences $\left\{\tilde{x}_{n}\right\}$ and $\left\{\tilde{y}_{n}\right\}$ are bounded, and Proposition [3.5. Theorem 3.15, and Theorem 3.16] show that they converge to zero weakly. Therefore

$$
\lim _{n \rightarrow \infty} \operatorname{Re}\left\langle R \tilde{x}_{n}, R i \tilde{y}_{n}\right\rangle=0 .
$$

However,

$$
\operatorname{Re}\left\langle R \tilde{x}_{n}, R i \tilde{y}_{n}\right\rangle=2^{n}\left\langle 1_{\left(0,2^{-n}\right]} \otimes f, 1_{\left(0,2^{-n}\right]} \otimes L f\right\rangle=\lambda,
$$

which is a contradiction. Therefore we get $L=0$. This shows that the restriction of $R$ to $G_{0, t}$ is an equivalence operator and finally we finish the proof.

Let $R_{t}$ be the restriction of $R$ to $G_{0, t}$ in the above proof. Then $V_{t}=\Gamma\left(R_{t}\right)$ and

$$
R i^{t} y(p)_{r, s}^{\prime}=i\left(R_{t}^{*}\right)^{-1 t} y(p)_{r, s}^{\prime} \text {. }
$$

For any positive invertible operator $D \in \mathbb{B}(\mathcal{K})$, we denote by $J^{0}(D)_{t}$ the linear extension of the map

$$
G_{0, t}^{0} \ni x(p)_{r, s} \mapsto{ }^{t} y(D p)_{r, s}^{\prime} \in G_{0, t}^{0}{ }^{\prime} .
$$

When $J^{0}(D)_{t, 0}$ has a bounded extension to $G_{0, t}$, we denote it by $J(D)_{t}$. For $D=1$, we have

$$
i\left(R_{t}^{*}\right)^{-1} J^{0}(1)_{t} x(p)_{r, s}=1_{(r, s]} \otimes \eta(p)=1_{(r, s]} \otimes \eta \xi^{*}\left(\xi^{*}\right)^{-1} p=i 1_{(r, s]} \otimes\left(\xi^{*}\right)^{-1} p,
$$

and so

$$
J^{0}(1)_{t, 0} x(p)_{r, s}=R_{t}^{*} 1_{(r, s]} \otimes\left(\xi^{*}\right)^{-1} p=R_{t}^{*}\left(1 \otimes\left(\xi \xi^{*}\right)^{-1}\right) R_{t} x(p)_{r, s} .
$$

Therefore when the resulting product system is of type I, the operator $J^{0}(1)_{t}$ always has a bounded extension $J(1)_{t}=R_{t}^{*}\left(1 \otimes\left(\xi \xi^{*}\right)^{-1}\right) R_{t}$. A similar computation shows $J\left(\xi^{*} \xi\right)_{t}=R_{t}^{*} R_{t}$, which is an equivalence operator.

Theorem 4.4. Let $\left(\left\{G_{a, b}\right\},\left\{S_{t}\right\}\right)$ be a sum system. Then the following statements are equivalent.

(1) The product system $\left(H_{t}, U_{s, t}\right)$ arising from $\left(\left\{G_{a, b}\right\},\left\{S_{t}\right\}\right)$ is of type $I$.

(2) There exists a positive invertible operator $D \in \mathbb{B}(\mathcal{K})$ such that the operator $J^{0}(D)_{t}$ extends to a bounded invertible operator $J(D)_{t}$ on $G_{0, t}$ for all $t>0$ such that $J(D)_{t} \in \mathcal{S}\left(G_{0, t}, G_{0, t}\right)$. 
(3) There exists a positive invertible operator $D \in \mathbb{B}(\mathcal{K})$ such that the operator $J^{0}(D)_{1}$ extends to a bounded operator $J(D)_{1}$ on $G_{0,1}$ such that $J(D)_{1} \in$ $\mathcal{S}\left(G_{0,1}, G_{0,1}\right)$.

Proof. We have already seen that (1) implies (2). The implication from (2) to (3) is trivial. The proof of the implication from (3) to (1) is the same as the proof of [8, Theorem 4.9].

Remark 4.5. Let the notation be as above. We set $F_{0, t}=\operatorname{Ker}\left(S_{t}^{*}\right)$ and $F_{s, t}=$ $T_{s} F_{0, t-s}$. Then $\left(\left\{F_{a, b}\right\},\left\{T_{t}\right\}\right)$ is also a sum system giving rise to a generalized CCR flow cocycle conjugate to that for $\left(\left\{G_{a, b}\right\},\left\{S_{t}\right\}\right)$. When the index is one, Theorem 4.4 shows that these two sum systems are isomorphic if and only if the resulting product system is of type I. This means that when the resulting product system is of type III, the two sum systems above are not isomorphic though they give cocycle conjugate generalized CCR flows.

\section{Perturbations of the Shift}

Let $K_{\mathbb{R}}$ be a real Hilbert space, and let $\left\{S_{t}\right\}$ be the shift semigroup of $L^{2}\left((0, \infty), K_{\mathbb{R}}\right)$. When $\operatorname{dim} K_{\mathbb{R}}=1$, we gave a complete characterization of the perturbations $\left\{T_{t}\right\}$ of $\left\{S_{t}\right\}$ in terms of analytic functions on the right half-plane in [7, and the index of the pair $\left(\left\{S_{t}\right\},\left\{T_{t}\right\}\right)$ is always 1. Here we take the first step to generalize our analysis to the case with non-trivial multiplicity space $K_{\mathbb{R}}$.

Theorem 5.1. Let $\left\{S_{t}\right\}$ be the shift semigroup of $L^{2}\left((0, \infty), K_{\mathbb{R}}\right)$. Then the index of any perturbation pair $\left(\left\{S_{t}\right\},\left\{T_{t}\right\}\right)$ of $C_{0}$-semigroups is $\operatorname{dim} K_{\mathbb{R}}$.

Proof. Let $A$ and $B$ be the generators of $\left\{S_{t}\right\}$ and $\left\{T_{t}\right\}$, respectively. The operator $A$ is the differential operator $A f=-f^{\prime}$ with the domain $D(A)$ consisting of all locally absolutely continuous $K_{\mathbb{R}}$-valued functions $f$ on $[0, \infty)$ such that $f^{\prime} \in L^{2}\left((0, \infty), K_{\mathbb{R}}\right)$ and $f(0)=0$. The adjoint operator $A^{*}$ is the differential operator $A f=f^{\prime}$ without boundary condition. Note that $B$ is a restriction of $-A^{*}$. We use the same notion as in Section 3. Recall that $\mathcal{K}$ is the orthogonal complement of $D(B)$ in $D\left(A^{*}\right)$ with respect to the graph inner product $\langle\cdot, \cdot\rangle_{A^{*}}$. By definition, all we have to show is $\operatorname{dim} \mathcal{K}=\operatorname{dim} K_{\mathbb{R}}$.

Let

$$
C=\lim _{t \rightarrow+\infty} \frac{\log \left\|T_{t}\right\|}{t} .
$$

Then every real number $s>C$ belongs to the resolvent set of $B$. For such an $s$, we introduce a linear map $M(s)$ from $D\left(A^{*}\right)$ to $K_{\mathbb{R}}$ by setting

$$
M(s) f=\int_{0}^{\infty} e^{-s x} f(x) d x-s \int_{0}^{\infty} e^{-s x} f^{\prime}(x) d x .
$$

Note that if $\xi \in K_{\mathbb{R}}$ is in the orthogonal complement of $\{M(s) p ; p \in \mathcal{K}\}$, then the function $e^{-s x} \xi$ belongs to the domain of $B$ and it is an eigenvector of $B$ with eigenvalue $s$. This implies $\operatorname{dim} K_{\mathbb{R}} \leq \operatorname{dim} \mathcal{K}$. Indeed, if $\mathcal{K}$ were finite dimensional and $\operatorname{dim} \mathcal{K}$ were strictly smaller than $\operatorname{dim} K_{\mathbb{R}}$, every $s>C$ would belong to the spectrum of $B$, which is a contradiction.

Suppose now that $\operatorname{dim} \mathcal{K}$ is strictly greater than $\operatorname{dim} K_{\mathbb{R}}$. We fix $s>C$. Then there would exist $p \in \mathcal{K} \backslash\{0\}$ such that $M(s) p=0$. Take $f \in L^{2}\left((0, \infty), K_{\mathbb{R}}\right)$ and 
set $g=(s I-B)^{-1} f$. Solving the differential equation $g^{\prime}+s g=f$, we get

$$
g(x)=e^{-s x} g(0)+\int_{0}^{x} e^{s(t-x)} f(t) d t
$$

Since $g \in D(B)$, we have

$$
\begin{aligned}
0 & =\langle p, g\rangle+\left\langle p^{\prime}, g^{\prime}\right\rangle=\langle p, g\rangle+\left\langle p^{\prime}, f-s g\right\rangle=\left\langle p^{\prime}, f\right\rangle+\left\langle p-s p^{\prime}, g\right\rangle \\
& =\left\langle p^{\prime}, f\right\rangle+\int_{0}^{\infty}\left\langle p(x)-s p^{\prime}(x), e^{-s x} g(0)+\int_{0}^{x} e^{s(t-x)} f(t) d t\right\rangle d x \\
& =\left\langle p^{\prime}, f\right\rangle+\langle M(s) p, g(0)\rangle+\int_{0}^{\infty} \int_{0}^{x} e^{s(t-x)}\left\langle p(x)-s p^{\prime}(x), f(t)\right\rangle d t d x \\
& =\left\langle p^{\prime}, f\right\rangle+\int_{0}^{\infty} \int_{0}^{\infty} e^{-s y}\left\langle p(y+t)-s p^{\prime}(y+t), f(t)\right\rangle d y d t \\
& =\left\langle p^{\prime}, f\right\rangle+\left\langle\left(s I-A^{*}\right)^{-1}\left(p-s p^{\prime}\right), f\right\rangle .
\end{aligned}
$$

Since $f$ is arbitrary, we get $p^{\prime}+\left(s I-A^{*}\right)^{-1}\left(p-s p^{\prime}\right)=0$. This implies $p^{\prime} \in D\left(A^{*}\right)$ and $s p^{\prime}-p^{\prime \prime}+p-s p^{\prime}=0$, and so $p^{\prime \prime}=p$. Since $p \in L^{2}\left((0, \infty), K_{\mathbb{R}}\right)$ and $p \neq 0$, this is possible only if $p(x)=e^{-x} \xi$ for some $\xi \in K_{\mathbb{R}} \backslash\{0\}$. However, this $p$ does not satisfy $M(s) p=0$, which is a contradiction, and we conclude $\operatorname{dim} \mathcal{K}=\operatorname{dim} K_{\mathbb{R}}$.

\section{ACKNOWLEDGEMENT}

The author would like to thank R. Srinivasan for useful discussions.

\section{REFERENCES}

[1] H. Araki, On quasifree states of the canonical commutation relations. II. Publ. Res. Inst. Math. Sci. 7 (1971/72), 121-152. MR0313834(47:2388)

[2] W. Arveson, Continuous analogues of Fock space, Mem. Amer. Math. Soc. 80(409):1-66, 1989. MR987590 (90f:47061)

[3] W. Arveson, Continuous analogues of Fock space. IV. Essential states, Acta Math. 164 (3/4) 265-300, 1990. MR1049159 (91d:46074)

[4] W. Arveson, Noncommutative dynamics and E-semigroups, Springer Monographs in Math, Springer, 2003. MR1978577 (2004g:46082)

[5] B. V. R. Bhat and R. Srinivasan, On product systems arising from sum systems, Infinite dimensional analysis, quantum probability and related topics, Vol. 8, Number 1, March 2005. MR2126876 (2006e:46075)

[6] A. van Daele, Quasi-equivalence of quasi-free states on the Weyl algebra. Comm. Math. Phys. 21 (1971), 171-191. MR0287844 (44:5046)

[7] M. Izumi, A perturbation problem for the shift semigroup. J. Funct. Anal. 251, (2007), 498545. MR2356422

[8] M. Izumi and R. Srinivasan, Generalized CCR flows. Commun. Math. Phys. 281, (2008), 529-571. MR2410905

[9] V. Liebscher, Random sets and invariants for (type II) continuous product systems of Hilbert spaces, Preprint math.PR/0306365.

[10] K. R. Parthasarathy, An Introduction to Quantum Stochastic Calculus, Birkhäuser Basel, Boston, Berlin (1992). MR1164866 (93g:81062)

[11] R. T. Powers, A nonspatial continuous semigroup of $*$-endomorphisms of $\mathfrak{B}(\mathfrak{H})$, Publ. Res. Inst. Math. Sci. 23 (1987), 1053-1069. MR935715 (89f:46118)

[12] M. Skeide, Existence of $E_{0}$-semigroups for Arveson systems: Making two proofs into one. Infin. Dimens. Anal. Quantum Probab. Relat. Top. 9 (2006), 373-378. MR2256500 (2007e:46057) 
[13] B. Tsirelson, Non-isomorphic product systems. Advances in Quantum Dynamics (South Hadley, MA, 2002), 273-328, Contemp. Math., 335, Amer. Math. Soc., Providence, RI, 2003. MR2029632 (2005b:46149)

[14] K. Yosida, Functional Analysis. Sixth edition. Springer-Verlag, Berlin-New York, 1980. MR617913 (82i:46002)

Department of Mathematics, Kyoto University, Kyoto, Japan

E-mail address: izumi@math.kyoto-u.ac.jp 